\title{
A possible resolution of the strange quark polarization puzzle?
}

\author{
Elliot Leader \\ Imperial College London \\ Prince Consort Road, London SWr $2 B W$, England \\ Alexander V. Sidorov \\ Bogoliubov Theoretical Laboratory \\ Joint Institute for Nuclear Research, 141980 Dubna, Russia \\ Dimiter B. Stamenov \\ Institute for Nuclear Research and Nuclear Energy \\ Bulgarian Academy of Sciences \\ Blvd. Tsarigradsko Chaussee 72, Sofia 1784, Bulgaria
}

\begin{abstract}
The strange quark polarization puzzle, i.e. the contradiction between the negative polarized strange quark density obtained from analyses of inclusive DIS data and the positive values obtained from combined analyses of inclusive and semi-inclusive SIDIS data using de Florian et. al. (DSS) fragmentation functions, is discussed. To this end the results of a new combined NLO QCD analysis of the polarized inclusive and semi-inclusive DIS data, using the Hirai et. al. (HKNS) fragmentation functions, are presented. It is demonstrated that the polarized strange quark density is very sensitive to the kaon fragmentation functions, and if the set of HKNS fragmentation functions is used, the polarized strange quark density obtained from the combined analysis turns out to be negative and well consistent with values obtained from the pure DIS analyses.
\end{abstract}

PACS numbers: 13.60.Hb, 12.38.-t, 14.20.Dh 
In the absence of neutrino reactions on a polarized target, the inclusive polarized deep inelastic lepton-hadron reactions determine only the sum of quark and anti-quark polarized parton density functions (PDFs), $\Delta q(x)+\Delta \bar{q}(x)$, and provide no information at all about the individual polarized anti-quark densities. All analyses of the polarized inclusive (DIS) data have produced results for the polarized strange quark density function, $\Delta s(x)+\Delta \bar{s}(x)$, which are significantly negative for all values of $x$ (for more recent analyses see [1, 2]). One way to determine polarized quark and anti-quark densities separately is to use the data on polarized semi-inclusive reactions (SIDIS) like $l+p \rightarrow l+h+X$, where $h$ is a detected hadron. In the past few years more data on polarized SIDIS processes have become available and have led to assertions that $\Delta s(x)+\Delta \bar{s}(x)$ is positive for most of the range of measured $x$. In the following we discuss possible resolutions to this puzzling state of affairs.

It should be noted that in the study of the SIDIS data it is usual to simplify the analysis by taking $\Delta s(x)=\Delta \bar{s}(x)$. On the other hand, it has been suggested, on the basis of theoretical models, that this equality is badly broken [3], and that this could be the cause of the conflict. However, it is crucial to realize that: (i) the DIS result for $\Delta s(x)+\Delta \bar{s}(x)$ is independent of the relationship between $\Delta s(x)$ and $\Delta \bar{s}(x)$, and (ii) that the COMPASS estimate [4] of the difference $\Delta s(x)-\Delta \bar{s}(x)$ is much smaller than the theoretical model estimates and thus cannot be the cause of a serious error in the extraction of $\Delta s(x)+\Delta \bar{s}(x)$ from a combined analysis of the DIS and SIDIS data.

The key to resolving the puzzle lies, we believe, in the properties of the fragmentation functions (FFs) needed in the theoretical expressions for the measured SIDIS cross-sections and asymmetries, which involve convolutions of either unpolarized or polarized PDFs with the FFs. There are three modern versions of the FFs in the literature, Hirai et al. (HKNS) [5], de Florian et al. (DSS) [6] and Albino et. al. $(\mathrm{AKK})$ [7], sometimes differing significantly from each other. They are based mainly on semi-inclusive $e^{+} e^{-}$annihilation data (HKNS), $e^{+} e^{-}$annihilation and RHIC data on reactions like $p p \rightarrow \pi$ or $K+X(\mathrm{AKK})$, and a global analysis (DSS) of the data on semi-inclusive $e^{+} e^{-}$annihilation, the proton-proton collisions at RHIC and unpolarized SIDIS processes.

The early claim by the HERMES Collaboration [8] that the polarized SIDIS data implied marginally positive $\Delta s(x)+\Delta \bar{s}(x)$ in the measured $x$ range [0.023-0.3] was based on a LO analysis of the data. In 2008, de Florian, Sassot, Stratmann and Vogelsang (DSSV) carried out a combined NLO QCD analysis [9] of polarized DIS, SIDIS and RHIC data using the DSS fragmentation functions and effectively confirmed the LO result. More precisely, using the assumption $\Delta s(x)=\Delta \bar{s}(x)$ they obtained a signchanging solution for $\Delta s(x)+\Delta \bar{s}(x)$, negative for $x<0.03$ and positive in the region $x>0.03$. Later we repeated this analysis [10], using polarized DIS and SIDIS data and found substantial agreement with DSSV. We confirmed the sign changing behavior of $\Delta \bar{s}(x)$, though our $\Delta \bar{s}(x)$ is less negative at $x<0.03$ and less positive for large $\mathrm{x}$ and compatible with zero within the errors. Note that the polarized pp data from RHIC are not important for the determination of the polarized quark and anti-quark densities; 
they constrain mainly the gluon polarization.

After convincing ourselves that the puzzle could not be resolved by taking $\Delta s(x) \neq$ $\Delta \bar{s}(x)$ we began to try to test whether the problem lay in the properties of the FFs. Now the largest disagreements between the various sets of FFs in the literature occur in the kaon production sector. To this end we first carried out a combined NLO QCD analysis [11] of the polarized world DIS data [12] and just the pion SIDIS data [4, 13], using the DSS FFs. Note that in this case only the sum $x(\Delta s+\Delta \bar{s})\left(x, Q^{2}\right)$ can be determined from the data because of the reasonable assumption $D_{s}^{\pi}=D_{\bar{s}}^{\pi}$ used for all the sets of the fragmentation functions. The result for $x(\Delta s+\Delta \bar{s}) / 2$ is illustrated in Fig. 1 and compared to those obtained from the LSS'06 DIS analysis [2] (red curve) and the combined LSS'10 fit to the DIS and SIDIS data [10] (black curve). As seen from Fig. 1, in the presence of only the $A_{1 N}^{\pi}$ data, $x(\Delta s(x)+\Delta \bar{s}(x)) / 2$ (blue curve) is still negative in the measured $x$ region as in the analyses of the purely inclusive data.

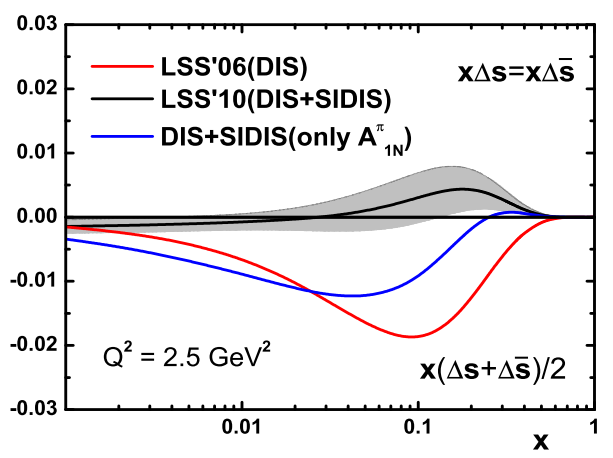

Figure 1: Comparison between polarized strange quark densities obtained from different kinds of NLO QCD analyses (see the text).

This definitely seemed to point towards the kaon FFs as the source of the conflict. Note also that it had already been pointed out by COMPASS Collaboration (2nd and 3rd refs in [13]) that in the LO QCD approximation the value of the first moment of $\Delta s(x)$ in the measured range of $x$ is very sensitive to the assumed value of the ratio of the $\bar{s}$-quark to $u$-quark fragmentation functions into positive kaons. Therefore, we carried out a new combined NLO QCD analysis of the polarized DIS and all the SIDIS data [4, 13, 14] using the HKNS set of FFs [5], which differ significantly from the DSS ones in the kaon sector, especially for the transition $\bar{s} \rightarrow K^{+}$, as shown in Fig 2 [15]. In Fig. 2 two error bands for the HKNS FFs are presented. The narrow one corresponds to $\Delta \chi^{2}=1$ while the wide corridor corresponds to $\Delta \chi^{2}=19.2$. The latter value corresponds to 17 parameters fit in the MINUIT-procedure when only the statistical errors are taken into account. However, the authors of [5] apply this procedure for the statistical and systematic errors added in quadrature which definitely overestimates 
the uncertainties.
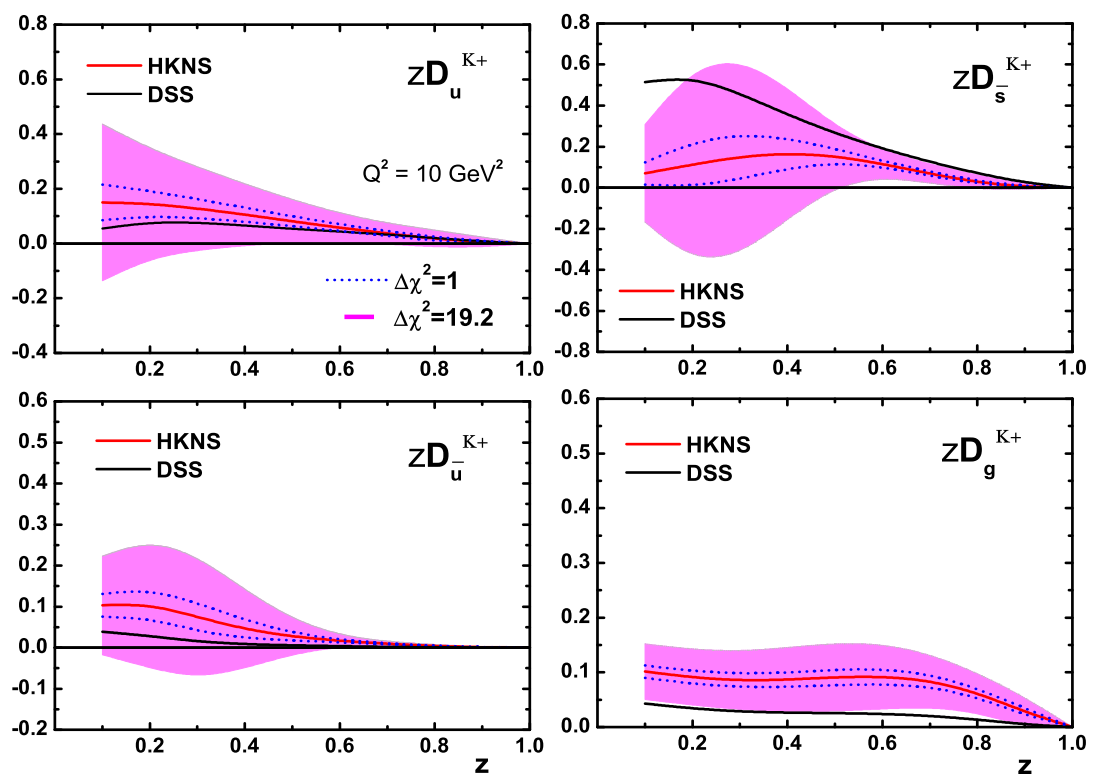

Figure 2: Comparison between NLO HKNS and DSS kaon FFs at $Q^{2}=10 \mathrm{GeV}^{2}$.

The method used is the same as in our previous analysis [10] of the same set of data when the DSS FFs were used. Note that the present SIDIS data are not precise enough to determine separately $\Delta s(x)$ and $\Delta \bar{s}(x)$. So, as in our previous analysis the assumption $\Delta s(x)=\Delta \bar{s}(x)$ was used. A good description of the SIDIS data $\left(\chi_{N r P}^{2}=0.92\right)$ is achieved using the HKNS FFs (NrP is the number of corresponding experimental points). The quality of the fit to the data is demonstrated in Fig. 3 (black curves) for some of the SIDIS asymmetries obtained by the HERMES and COMPASS Collaborations. The new curves are compared to our previous theoretical curves (red ones) obtained from the best fit to the data using the DSS FFs $\left(\chi_{N r P}^{2}=0.87\right)$. As seen from Fig. 3 the results from both the fits are very close to each other and for some of the asymmetries the curves are almost identical.

Let us discuss the impact of the HKNS fragmentation functions on the polarized sea-quark densities. It is known that the present SIDIS data do not influence the gluon polarization. It is mainly determined from inclusive DIS and semi-inclusive $p p$ RHIC data. The new values of the sea quark and gluon polarized densities (black curves) are presented in Fig. 4 together with their error bands and compared to those obtained using the DSS FFs (LSS'10). As seen from Fig. 4 the changes in the polarized sea quark densities are as follows: negligible for $x \Delta \bar{d}(x)$, visible for $x \Delta \bar{u}(x)$ at $x>0.03$ 

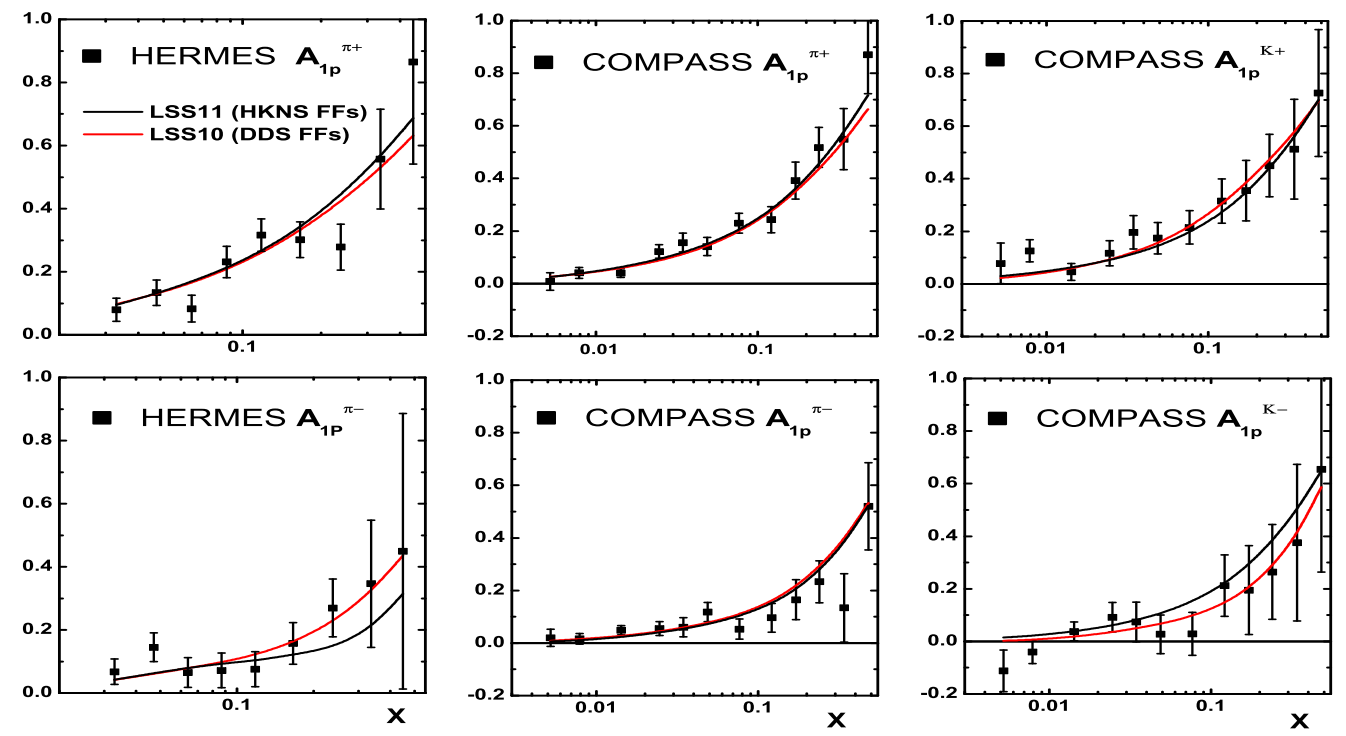

Figure 3: Comparison of our NLO LSS'11 (black curves) and LSS'10 (red curves) results for the SIDIS asymmetries with the data at measured $x$ and $Q^{2}$.

and dramatic for $x \Delta \bar{s}(x)$, although the central values of the first moments of $\Delta \bar{s}(\mathrm{DSS})$ and $\Delta \bar{s}(\mathrm{HKNS})$ are very close to each other $(-0.052 \pm 0.016$ and $-0.048 \pm 0.012$ at $Q^{2}=1 \mathrm{GeV}^{2}$ for DSS and HKNS FFs, respectively) and coincide within the errors. In Fig. 4 our LSS'06 result [2] for $x(\Delta s(x)+\Delta \bar{s}(x)) / 2$ (blue curve) obtained from the NLO QCD analysis of the world inclusive DIS data is presented too. We find now that if the HKNS FFs are used, $\Delta \bar{s}(x)$ is negative and well consistent with $(\Delta s(x)+\Delta \bar{s}(x)) / 2$ obtained from the pure DIS analyses [1, 2].

In conclusion, we have found that in the presence of semi-inclusive DIS data the strange quark density is very sensitive to the choice of the FFs. We have also demonstrated that the strange quark polarization puzzle can be resolved by using the HKNS set of fragmentation functions rather than the DSS ones. Finally, we like to stress we do not claim to have presented a unique resolution to the strange polarization puzzle. Our analysis illustrates only how badly we need to have a more reliable determination of FFs in order to extract correctly the polarized sea quark densities. To this end precise unpolarized SIDIS cross-section data are very important.

Acknowledgments: We thank S. Kumano and M. Hirai for the Fortran code of the HKNS fragmentation functions and some additional explanations. One of us (D. S) is also thankful to R. Sassot for useful discussions. This research was supported by the 

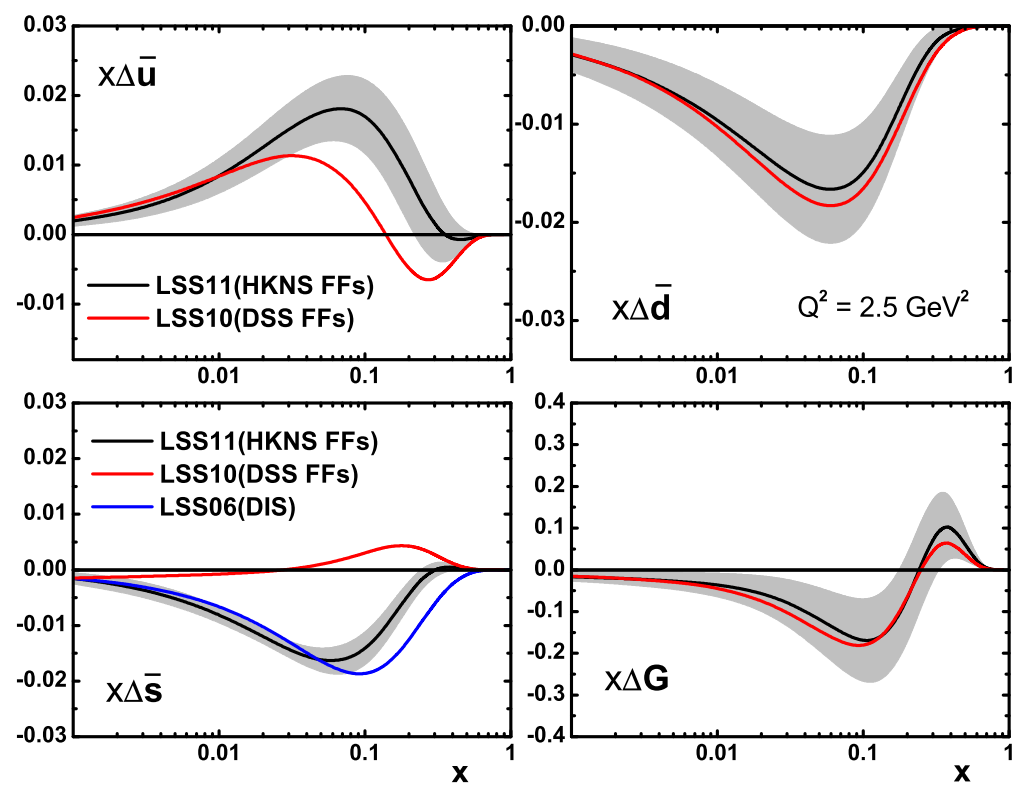

Figure 4: Comparison between NLO LSS'11(HKNS FFs) and LSS'10(DSS FFs) sea quark and gluon polarized PDFs at $Q^{2}=2.5 \mathrm{GeV}^{2}$. The blue curve corresponds to $x(\Delta s(x)+\Delta \bar{s}(x)) / 2$ obtained from the pure DIS analysis [2].

JINR-Bulgaria Collaborative Grant, by the RFBR Grants (No 09-02-01149 and 11-0100182) and by the Bulgarian National Science Fund under Contract 02-288/2008.

\section{References}

[1] M. Hirai, S. Kumano, and N. Saito (Asymmetry Analysis Collaboration), Phys. Rev. D 74, 014015 (2006); V.Y. Alexakhin et al. (COMPASS Collaboration), Phys. Lett. B 647, 8 (2007); J. Blumlein and H. Böttcher, Nucl. Phys. B841, 205 (2010).

[2] E. Leader, A.V. Sidorov, and D.B. Stamenov, Phys. Rev. D 75, 074027 (2007).

[3] S. J. Brodsky and Bo-Qiang Ma, Phys. Lett. B 381, 317 (1996); Y. Ding, RongGuang Xu, and Bo-Qiang Ma, Phys. Rev. D 71, 094014 (2005).

[4] M.G. Alekseev et al. (COMPASS Collaboration), Phys. Lett. B 693, 227 (2010).

[5] M. Hirai, S. Kumano, T.-H. Nagai, and K. Sudoh, Phys. Rev. D 75, 094009 (2007). 
[6] D. de Florian, R. Sassot, and M. Stratmann, Phys. Rev. D 75, 114010 (2007); Phys. Rev. D 76, 074033 (2007).

[7] S. Albino, B. A. Kniehl, and G. Kramer, Nucl. Phys. B 803, 42 (2008).

[8] U. Stosslein, Acta Phys. Polonica B 33, 2813 (2002); A. Airapetian et al. (HERMES Collaboration), Phys. Rev. Lett. 92, 012005 (2004).

[9] D. de Florian, R. Sassot, M. Stratmann, and W. Vogelsang, Phys. Rev. D 80, 034030 (2009).

[10] E. Leader, A.V. Sidorov, and D.B. Stamenov, Phys. Rev. D 82, 114018 (2010).

[11] E. Leader, A.V. Sidorov, and D.B. Stamenov, arXiv:1012.5033 [hep-ph] (to be published in Eur. Phys. J. ST).

[12] J. Ashman et al. (EMC Collaboration), Phys. Lett. B 206, 364 (1988); Nucl. Phys. B328, 1 (1989); P.L. Anthony et al. (SLAC E142 Collaboration), Phys. Rev. D 54, 6620 (1996); K. Abe et al. (SLAC E143 Collaboration), Phys. Rev. D 58, 112003 (1998); K. Abe et al. (SLAC/E154 Collaboration), Phys. Rev. Lett. 79, 26 (1997); P.L. Anthony et al. (SLAC E155 Collaboration), Phys. Lett. B 493, 19 (2000); Phys. Lett. B 463, 339 (1999); A. Airapetian et al. (HERMES Collaboration), Phys. Rev. D 71, 012003 (2005); X. Zheng et al. (JLab/Hall A Collaboration), Phys. Rev. Lett. 92, 012004 (2004); Phys. Rev. C 70, 065207 (2004); K.V. Dharmwardane et al. (CLAS Collaboration), Phys. Lett. B 641, 11 (2006); B. Adeva et al. (SMC Collaboration), Phys. Rev. D 58, 112001 (1998); V.Yu. Alexakhin et al. (COMPASS Collaboration), Phys. Lett. B 647, 8 (2007); M.G. Alekseev et al. (COMPASS Collaboration), Phys. Lett. B 690, 466 (2010).

[13] A. Airapetian et al. (HERMES Collaboration), Phys. Rev. D 71, 012003 (2005); M.G. Alekseev et al. (COMPASS Collaboration), Phys. Lett. B 680, 217 (2009); Phys. Lett. B 690, 466 (2010).

[14] B. Adeva et al. (SMC Collaboration), Phys. Lett. B 420, 180 (1998); M.G. Alekseev et al. (COMPASS Collaboration), Phys. Lett. B 660, 458 (2008).

[15] The significant difference in the kaon sector between the DSS FFs and the other sets of FFs, including HKNS, is due to the unpublished HERMES'05 data on the unpolarized hadron multiplicities used only in the DSS analysis. We consider that until the final HERMES and COMPASS data on the hadron multiplicities are presented, the question of a more reliable determination of the fragmentation functions in the $Q^{2}$ range of the present unpolarized SIDIS processes is open. 